\title{
EVOLUTION OF MAINTENANCE SYSTEMS OF PASSENGER AND FREIGHT WAGONS FROM THE ECM CERTIFICATION POINT OF VIEW
}

\begin{abstract}
RCM and ECM are abbreviations seemingly the same though the meaning is different. However, they have lot of common as the both are aimed at increased reliability of operation and minimising risk. RCM stands for Reliability Centred Maintenance and ECM stands for Entity in Charge of Maintenance, thus maintenance is the common term for both. Indeed, maintenance is a key for increased safety and reduced risk. The paper presents basic principles and theory behind the RCM - an advanced maintenance concept with its origin in aviation and consequently applied in industries connected with high level of risk, such power generation, etc. The ECM certification is a new approach for assessment of how railway vehicles operators are managing the risks connected with operation of the rolling stock. Entities, either owners or keepers of the rolling stock, are those who are responsible for the technical condition guaranteeing safety of railway traffic. There is common European methodology (requirements) for assessing the competence of ECMs based on EC Regulation No. 445/2011. Behind this methodology are efforts to minimise risks of freight trains operation and prevent serious accidents resulting from improper maintenance and insufficient technical state. In line with these efforts is our proposal for implementation of RCM in railway vehicles maintenance.
\end{abstract}

Keywords: Railway wagon, maintenance, risk, RCM, FMEA, ECM certification.

\section{Introduction}

Quality, safety and environment - these words stand for the basic requirements for any industrial and service activity, railway transport not excluding. There are few words that always go together - reliability, safety, risk, which characterise any system functioning. There are many factors influencing the reliability - the fundamental is so called inherent reliability - built in the product (system, machine) by its design and manufacture. Yet there are many more factors influencing the reliability of a system during its life (operation), among which perhaps the most important is maintenance. Inherent reliability is recognised even in maintenance terminology standard [1], which also recognises failure criticality as combination of failure consequence severity and its frequency (probability of occurrence). This is just for illustration that importance of risks is emphasised even in basic terminology standards.

Of course, problems of risk require much more than a basic definition. There are many methods dealing with risks analysis and their mitigation. In machines operation and maintenance risks are always connected with failures. In traditional maintenance approach the requirements for maintenance of each component were determined in accordance with its actual or assumed technical characteristics, without considering its failure consequences. The resulting maintenance schedules were used to all similar objects without considering the different consequences coming from different operational contexts.

Wherever possible, new approaches should be searched for that would synthesize new findings into a form so that it would be possible to evaluate and implement those approaches having the best value for functioning of physical objects and the whole organisation (company). Philosophy that provides such a framework is called Reliability Centred Maintenance (RCM) [2]. RCM uses analytical methods of a-priori reliability, especially:

1. Fault Tree Analysis (FTA),

2. Reliability Block Diagram (RBD),

3. Failure Mode and Effect Analysis (FMEA),

4. Root Cause Failure Analysis (RCFA).

These methods in broader context contribute also to Fault Prevention, Fault Forecasting, Fault Removal, and Fault Tolerance.

The area of fault forecasting is decisive from the maintenance system proposal point of view. RCM is a strong method in defining failure consequences (in fact it is recognition that the only reason for doing any form of preventive maintenance is not in preventing occurrence of failure themselves but in avoiding

\footnotetext{
* Peter Zvolensky, Vladimir Stuchly, Juraj Grencik, Roman Poprocky

Department of Transport and Handling Machines, Faculty of Mechanical Engineering, University of Zilina, Slovakia

E-mail: peter.zvolensky@fstroj.uniza.sk
} 
or at least in minimising failure consequences). However, for determination of intervals to maintenance action (maintenance tasks schedule) it is necessary to utilise the reliability methods.

\section{Maintenance of railway wagons}

\subsection{Current state of railway personal wagons maintenance system}

Similarly to manufacture, the maintenance system (maintenance types and maintenance strategies) of railway wagons has been evolving within the years.

In Table 1 is an example of maintenance cycle of selected types of railway passenger wagons after the $5^{\text {th }}$ change that has been introduced since the $1^{\text {st }}$ April, 2001. The railway wagons are divided into maintenance groups according to the "repair" types. Corresponding repair levels, mileages (distance run between maintenance interventions) and maintenance cycles are given in Table 1.

In the current organisation of preventive maintenance of railway vehicles at the Slovak Railway Company (ZSSK) according to the valid maintenance regulations the main decision criterion is so called mileage (in $\mathrm{km}$ ) and for certain types of wagons also time periods. This approach is based on relatively long evolution and, in principle, by tradition at implementation of planned-preventive maintenance system.

Process of monitoring, preparing and delivering vehicles into maintenance (at different maintenance levels) has its characteristic steps and individual elements, which in modern maintenance concepts, and in particular with regards to costs priority, do not always mean economically suitable conditions of predetermined maintenance itself.

1. The typical characteristics of currently performed maintenance of passenger vehicles are [4]:

2. Elaborated performance standards, especially at higher maintenance levels.

3. Technological routine and periodicity of maintenance actions.

4. Specialisation of workplaces and technological procedures at higher maintenance levels.

5. High routine in monitoring of simple indicators for delivery into maintenance.

6. Maintenance is not performed in accordance with real technical conditions of a railway vehicle and differentially.

7. Operational utilisation (conditions) is not considered at railway wagons.

8. Often uselessly vast disassembly of important structural units significantly worsen their overall functional stability and negatively influence the vehicles reliability.

Regulation V66 - Maintenance groups for selected types of wagons [3]

Table 1

\begin{tabular}{|l|l|l|r|}
\hline Maint. group & Wagon type & Repair level & Mileage $(\mathrm{km})$ \\
\hline \multirow{3}{*}{ 1a } & Salon wagons - RIC regime & R1 - 12 months & 50000 \\
& & R2 - 48 months & 200000 \\
& & R3 & 400000 \\
& & R4 & 800000
\end{tabular}

Nw (R4) R1 R1R1R2 R1 R1R1R3 R1 R1R1R2 R1 R1R1 R4

$3 \mathrm{~b}$

Other wagons (except for wagons of group 1 and 2) -

R1 - 6 months

110000 inland regime for InterCity trains

R2 - 36 months

440000

R3

1320000

3960000

Nw (R4) R1R1R1 R2 R1R1R1 R2 R1R1R1 R3 R1R1R1 R2 R1R1R1 R2 R1R1R1 R3 etc.

Four axle wagons of inland regime with speed over120 $\mathrm{km} / \mathrm{h}$ (except for wagons of group 1,2 and 3)

R1 - 6 months
R2 - 48 months
R3
R4

100000

300000

900000

1800000

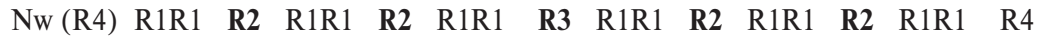

Legend: $\quad$ Nw - New Wagon

R1, R2, R3, R4 - Revisions (maintenance/repair level of 1 - 4) 
9. Fixed work of "hard" planned maintenance actions may result in ineffective labour costs and overheads.

10. Insufficient personal responsibility of workers for maintenance quality and, consequently, for technical condition of a railway vehicle.

11. In current financial situation at ZSSK, there is danger of not fulfilling the periodicity of maintenance actions given by regulations which deteriorates current technical condition of railway vehicles.

\subsection{Current state of railway freight wagons maintenance system}

In Slovakia, a generally accepted maintenance system for freight wagons is so called SUNV (Maintenance System of Freight Wagons; in Slovak: Systém údržby nákladných vozňov) based on the former Regulation V67 [5]. ZSSK Cargo and other freight wagons operators have the system of freight wagons periodic maintenance based on time considering a specific design of a freight wagon type.

The fundamental terms of the maintenance system are as follows:

1. "Medium repair N2.1, N2.2, N2.3, N4" - lower type of preventive periodic maintenance with due terms of 2 or 4 years. The basic scope of maintenance tasks is given in chapter 5 of the regulation. The main task is inspection and function test of components and functional subassemblies of freight wagons.

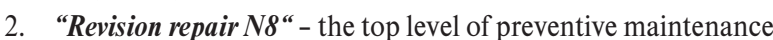
with due term of 8 years. The basic scope of maintenance tasks is given in chapter 5 of the regulation. The main task is revision and/or replacement of components and assemblies. There must always be a protocol issued on the regular technical check.

3. "Extended due term of periodic repair $+3 M^{\prime \prime}$ - extended operational period by 3 months after due term based on the technical inspection. This can be done only on approval of wagon's ECM (entity in charge of maintenance).

4. "Regular technical check" - technical check carried out in compliance with law. The main purpose is the check of actual technical state and assessment of technical condition before return to operation. Protocol on the technical check can be issued only by a competent person.

5. "Safety inspection" - technical inspection is carried out in the scope given in chapter 5 on freight wagons with 6 months delay after medium or revision repair due term or on wagons deposited more than 12 month for the purpose of transport into maintenance workshop or physical disposal.

From the analysis of forms, technology and consequences of current maintenance of railway wagons in Slovakia it can be concluded that keeping the current state is not realistic and economically probably unsustainable. That is why a system change aimed at rationalisation and improved maintenance effectiveness is necessary. Change of maintenance systems of railway vehicles must be based on their increased availability at more effective maintenance costs. Proposed transformation change is aimed at transition from current predetermined maintenance to predictive (reliability centred - condition based) maintenance.

\section{Utilisation of theory of reliability}

For utilisation of reliability characteristics in the maintenance of railway vehicles, the following questions should be answered:

1. How can the reliability characteristics be determined?

2. Is there a reason for their determination?

3. Is it reasonable to analyse elements or whole systems?

4. Which reliability characteristics are the most important for maintenance? And are some of them meaningful?

5. How, when, where and in what extent should the reliability characteristics be determined?

6. How can the calculations of reliability characteristics help in creation of maintenance systems?

Reliability of the already operated railway vehicles (because these are the most important for maintenance) was built-in during their design and manufacture phase (inherent reliability). Maintenance itself cannot increase these built-in reliability properties (but can preserve them).

Creation of maintenance system (based on the reliability theory) can be divided into the following steps:

1. Determination of reliability characteristics (by mathematicalstatistical methods).

2. Determination of optimum periods (intervals) of operation of components until the planned maintenance action. It is based on element's reliability and costs for planned (preventive) and unplanned (corrective) maintenance.

3. Creation of maintenance system. It is based on determined times (intervals) between maintenance for elements and costs of maintenance [6].

\subsection{Statistic and parametric reliability of elements}

In the reliability theory we look at failures in a mathematicalstatistical way as random phenomena because we are not able to reliably determine in advance the moment of occurrence of a failure and its extent because of complexity of phenomena related to the failure. Reliability analyses are carried out especially in the phase of concept definition, in the phase of designing and development and in the phase of utilisation (operation and maintenance) on different levels of structure breakdown for 
evaluation and estimation of reliability parameters of an item (element or system).

Stochastic reliability models can be determined from the detailed knowledge of behaviour of a given set of objects in operation. They must provide generalisation of obtained findings also on other similar items that were not directly subject of investigation.

Implementation of statistical methods needs the following subsystems:

1. System of information collection on operational reliability;

2. System of selection, use and primary analysis of evaluated sample of objects;

3. System of quantification of reliability indicators (parameters) of elements of objects;

4. System of quantification of object's reliability as a system;

5. System of consequent technical analysis and implementation of obtained knowledge in operation, maintenance etc.

The most suitable theoretical model that optimally characterises reliability of engineering products is a Weibull model in particular because in its generality it covers virtually most of possible function courses of random quantities of items reliability [7] and [8]. Weibull distribution function (three-parameter) is expressed by equation:

$F(t)=1-e^{-\left(\frac{t-c}{a}\right)^{b}}$, for $t>0$

where: $a>0$ is a scale parameter, $b>0$ is a shape parameter, $c$ is a location parameter

On this basis 5 fundamental reliability parameters (indicators) are defined: failure probability $Q(t)$, reliability probability $R(t)$, probability failure density $f(t)$, failure rate $\lambda(t)$, mean operation time to failure $T_{s t r}$. In Fig. 1 failure density and failure rate are illustrated.

Indicators of parametric reliability are the same as for statistic reliability and can be calculated in accordance with the selected model of parameter change. For calculations of indicators of parametric reliability it is necessary to determine equation of parameter change with initial value and a velocity of its change using a suitable method (Fig. 2).

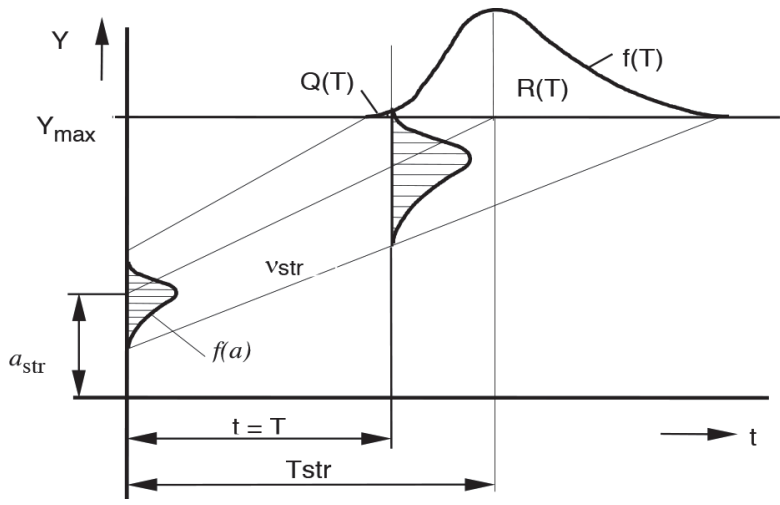

$$
\begin{aligned}
& Y(T)=a_{s t r}+k \cdot t \\
& R(T)=0.5+\Phi\left(\frac{Y_{\max }-a_{s t r}-v \cdot T}{\sigma_{a}}\right)
\end{aligned}
$$

where $\Phi$ is Laplace function

Fig. 2 Model of gradual failure formation (generalised) and calculation of reliability $R(T)$

\section{Optimisation model of maintenance intervals}

Time, or mileage, to repair can be determined for components reliability. To ensure required reliability level, planned preventive maintenance actions are carried out after a predetermined mileage $L(\mathrm{~km})$. For simplifying the mathematical model we assume that the duration of maintenance action is short compared to up-time (operation without failures).

Because of carrying out the maintenance, operation time of components will have a cut distribution $f(l)$ with mean value $L_{s t r}$. Value $L_{s t r}$ depends on the degree of cut, that is, the value of selected interval between maintenance $L$.

Probability of failure of a component during the interval $L(\mathrm{~km})$, which is the probability of corrective (unplanned) maintenance of the component, is

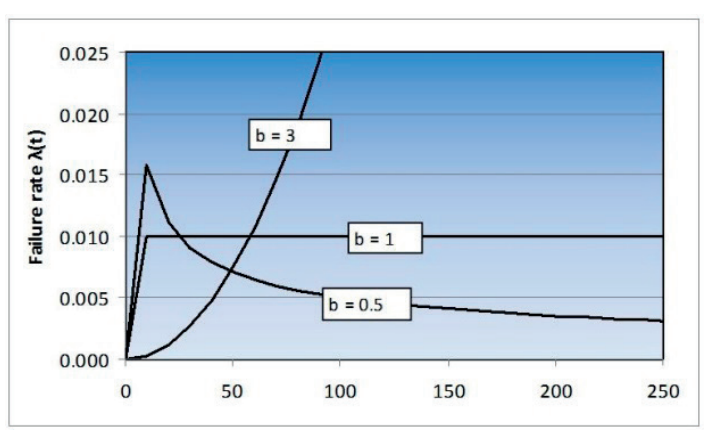

Fig. 1 Reliability parameters for Weibull distribution function for different $b$ 
$Q(L)=\int_{0}^{L} f(l) \mathrm{dl}$

The probability that a component is working without failure during $L(\mathrm{~km})$ and will be replaced (or restored) in planned maintenance is

$R(L)=1-Q(L)=1-\int_{0}^{L} f(l) \mathrm{dl}$

The task of determination of optimum interval between maintenance works leads to determination of such interval $L$ between planned (preventive) maintenance in which the total costs for keeping objects up-state will be minimum.

We will determine overall costs connected with restoring failed components of i-th subsystem during the interval $L^{\prime}(\mathrm{km})$; for this we will use the designation:

$C_{N}$ - mean value of i-th subsystem in corrective maintenance costs (besides direct costs for repair, the production and other induced losses should be included);

$C_{P}$ - mean value of costs of component of i-th subsystem in preventive maintenance.

Maintenance costs for intervals $L^{\prime}(\mathrm{km})$ will be calculated using the formula for calculation of $M$, that is the mean value of costs for restoring one component of i-th subsystem for the interval Lstr $(\mathrm{km})$ in the ratio of costs for preventive and corrective maintenance:

$M=\frac{\int_{0}^{\infty} R(l) \cdot \mathrm{dl}}{\int_{0}^{L} R(l) \cdot \mathrm{dl}} \cdot[1-(1-p) \cdot R(L)]$

where: $p=C_{P} / C_{N}-$ ratio of costs for preventive and corrective maintenance. Condition $C_{P} \leq C_{N}$ is assumed; then value of $p$ is within the interval $0<p \leq 1$.

From (4) it concludes that $M$ is a function of selected interval between maintenance $L$ and depends on the type and parameters of probability distribution function and also on ratio of costs $p$. For analysis of solution of function $M$ it is necessary to find analytical expression for various types (most commonly used) of failure probability distribution functions.

For Weibull distribution:

$$
M=\frac{\Gamma\left(1+\frac{1}{b}\right) \cdot\left[1-(1-p) \cdot e^{-\vartheta_{c}^{b}}\right]}{\int_{0}^{\frac{L}{a}} e^{-\vartheta_{c}^{b}} \cdot \mathrm{d} \vartheta_{e}}
$$

For analysis of the formula (5) we use a graphic interpretation of $M=f(\vartheta e, p, b)$ in Fig. 3 for $\mathrm{b}=3.43$ and for various values of $p$. Optimum interval for maintenance can be found at the minimum of the function $M$. From the minimum of function $M$ we obtain the value of optimum mileage (in km) multiplying $M$ by $a$ (scale parameter of Weibul distribution).

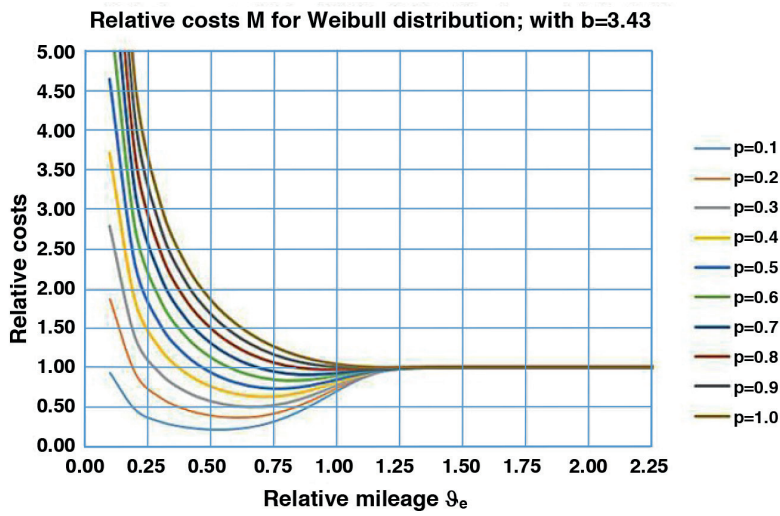

Fig. 3 Graph of relative costs $M$ for Weibull probability distribution with shape parameter $b=3.43$

\section{Reliability Centred Maintenance and ECM}

Because of inaccessibility of the data from operation of railway vehicles it is not possible to fully use the methods of a posteriori reliability for determination of maintenance tasks.

Both maintenance regulations for railway wagons follow the conventional maintenance systems. RCM method takes into consideration failure consequences and CONSEQUENCES are evaluated as RISK.

Complex definition of RCM is: "Process used to determine what must be done to ensure that any physical asset continues to do whatever its users want it to do in its present operating context" [2]. When evaluating consequences one of the decisive categories is RISK.

The railway undertakings or the infrastructure managers should ensure, through their safety management system, the control of all risks related to their activity, including the use of contractors. To this purpose, a railway undertaking should rely on contractual arrangements involving entities in charge of maintenance for all wagons it operates. This could be a contract between the railway undertaking and the entity in charge of maintenance or a chain of contracts involving other parties, such as the keeper. These contracts should be consistent with the procedures outlined by a railway undertaking or an infrastructure manager in its safety management system, including for the exchange of information [9].

Risk assessment is a structured approach to assess risks associated with the maintenance of freight wagons, including those directly arising from operational processes and the activities of other organisations or persons, and to identify the appropriate risk control measures. 


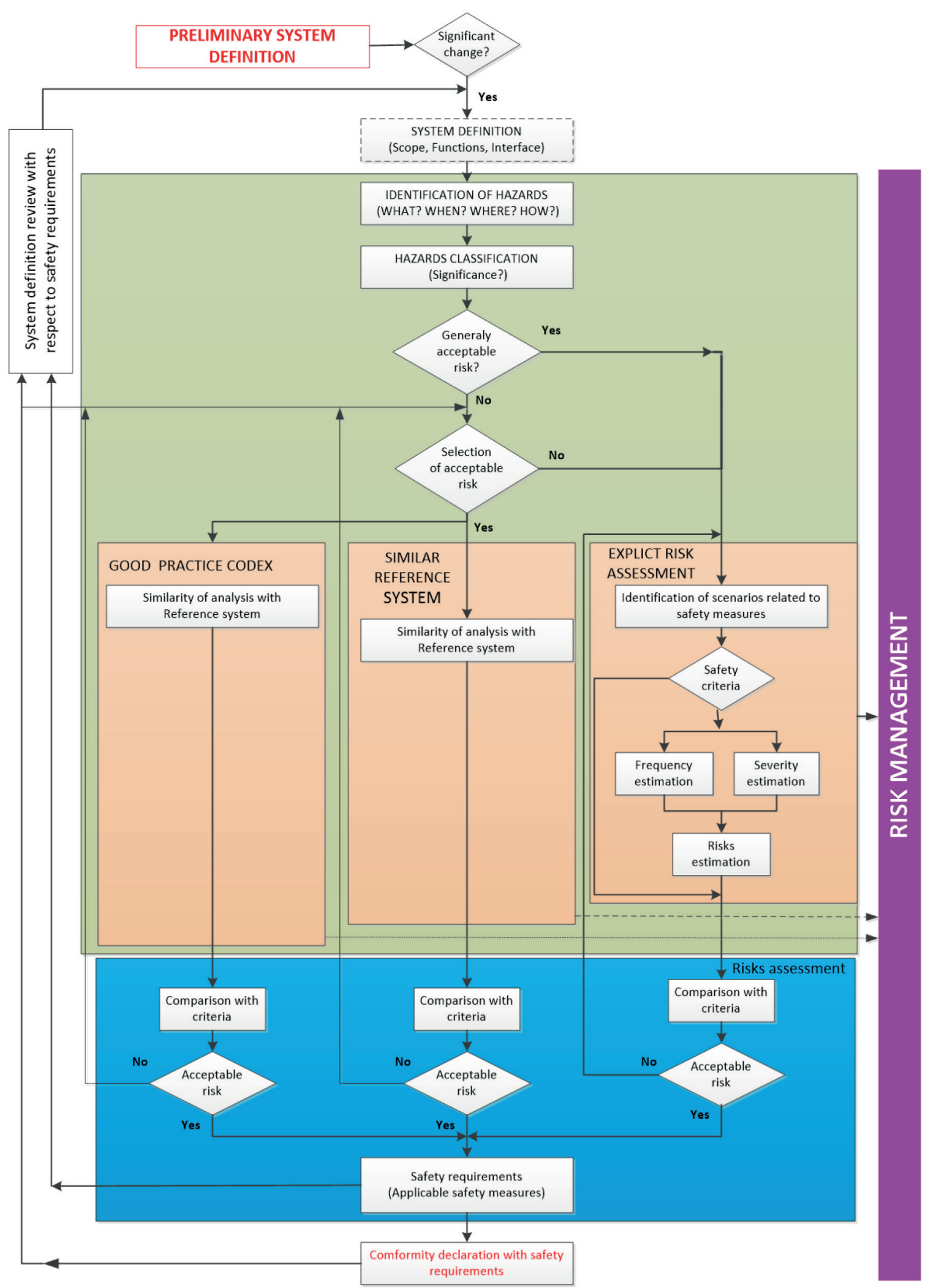

Fig. 4 System of risk assessment (adapted from [10]) 
Complex process of risk assessment is shown in Fig. 4. This process can be applied in general as well as for risk assessment of freight wagons [10].

The organisation must have procedures for:

1. analysing risks relevant to the extent of operations carried out by the organisation, including the risks arising from defects and construction non-conformities or malfunctions throughout the lifecycle;

2. evaluating the risks referred to in point (a);

3. developing and putting in place risk control measures.

The organisation must have a procedure to regularly collect, monitor and analyse relevant safety data, including:

1. the performance of relevant processes;

2. the results of processes (including all contracted services and products);

3. the effectiveness of risk control arrangements;

4. information on experience, malfunctions, defects and repairs arising from day-to-day operation and maintenance.

For solutions within RCM, a FMEA method (Failure Mode and Effect Analysis (FMEA) is used, which in RCM is formalised as RCM information and RCM decision form.

The FMEA is a systematic set of actions carried out with the purpose to:

1. identify and evaluate possible failure of a product/process and consequences of this failure,

2. determine measures that would reduce probability of creation (occurrence) of possible failure,
3. document the whole process.

It completes the process of definition of what the design or process for customer's satisfaction should fulfil.

The FMEA method represents a team work aimed at definition of possible failure modes and their consequences. Based on experience it is said that by this method it is possible to reveal $70-90 \%$ of failures. Base and purpose of FMEA is to exclude, or to acceptable level reduce failures of production processes and products during their utilisation by minimising the risk number $R$. Risk number $R$ is given by multiplication of failure occurrence probability ranking $\mathrm{Z}$, severity of failure consequences $\mathrm{V}$ and probability of preventive failure detection $\mathrm{D}$ :

$R=Z * V * D$

At the Department of Transport and Handling machines we have been using FMEA process supported by software tool IQ RM PRO 6. Structured risks of a freight wagon created by this program are shown in Fig. 5.

\section{Conclusions}

Trends to minimise risk in railway operation are among the most important topics in Europe. European commission through its regulation Commission Regulation (EU) No 445/2011 intends to improve safety of railway, especially freight transport

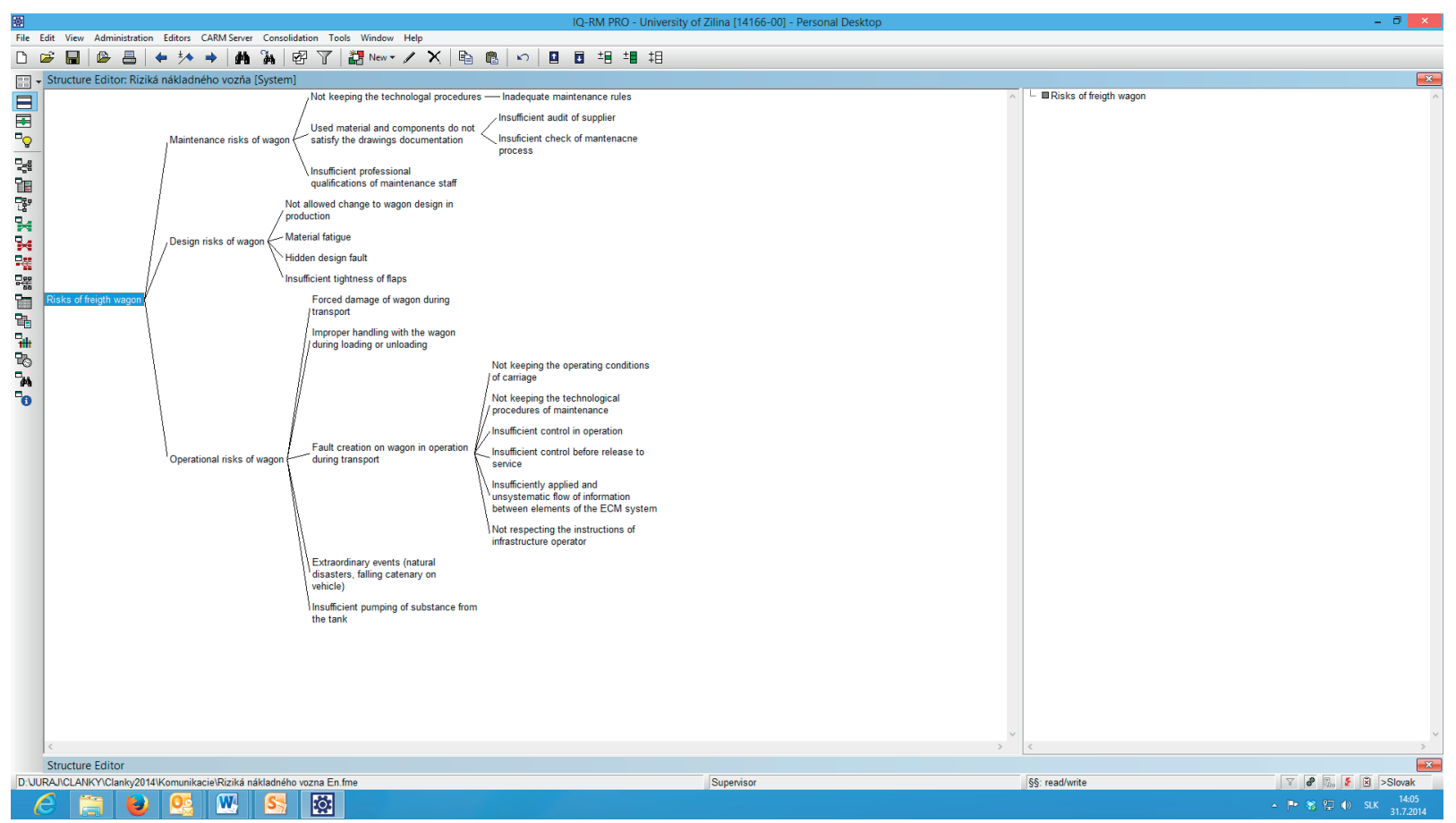

Fig. 5 Risks of a freight wagon (example) 
by creating system of certified ECMs (Entities in Charge of Maintenance) that are fully responsible for performing all maintenance functions properly. The Department of Transport and Handling Machines, University of Zilina, was recognised as an ECM certification body. It has audited and certified most of the railway freight wagons operators in Slovakia. Generally, it can be concluded that the certification process forced the rolling stock maintenance operators to review their approach to risks and to start new thinking of risk management.
Theoretical methods of risks assessment presented in the paper are base and tool for analysis in real practice and consequent improvement of operational safety of freight wagons. Freight wagons operators have to realise the importance of safe operation, though demanding more efforts, costs and enhancements in maintenance, but this should be balanced by reduced number of accidents. Consequences of accidents in railway freight transport are mostly very costly and their prevention is worth searching for improvements.

\section{References}

[1] STN EN 13306: Maintenance - Terminology (in Slovak), 2010.

[2] MOUBRAY, J.: Reliability-centered Maintenance, Industrial Press Inc : New York 1997, ISBN 0-8311-3078-4.

[3] ZSSK V 66: Railway Wagons Maintenance Regulation (in Czech), Praha : NADAS, 1976 (applicable from 1.7.1976 - modified 19.6.2001).

[4] STUCHLY, V., GRENCIK, J.: Proposed Implementation of RCM Method for Maintenance of Bogies of Railway Passenger Wagon, EuroMaintenance 2012, Belgrade, May, 2012, 87-96. ISBN 978-86-89141-00-9, CD-ROM.

[5] Kol.: Railway Wagons Maintenance Regulation (in Slovak), version 2.0, ZSSK Cargo Slovakia: Bratislava, 2011.

[6] STUCHLY, V., GRENCIK, J.: Calculation of Preventive Maintenance Tasks using RCM Method, EuroMaintenance 2010 Conference, May 2010, Fiera di Verona, 207- 209.

[7] STUCHLY, V., POPROCKY, R.: Maintainance Machine and Devices - $1^{\text {st }}$ ed. (in Slovak), Zilinska univerzita : Vysokoskolske ucebnice, 2014, ISBN 978-80-554-0845-3.

[8] ABERNETHY, R. B.: The New Weibull Handbook, Fifth Edition (Reliability \& Statistical Analysis for Prediction Life, Safety, Survivability, Risk, Cost a Warranty Claims - Waloddi Weibull). Published and distributed by Dr. Robert B. Abernethy, 536 Oyster Road, North Palm Beach, Florida 33408-4328, 2004, ISBN-10 0-9653062-3-2.

[9] Commission Regulation (EU) No. 445/2011 of 10 May 2011 on a system of certification of entities in charge of maintenance for freight wagons and amending Regulation (EC) No. 653/2077.

[10] ERA/GUI/02-2008/SAF, Version: 1.1: Collection of Examples of Risk Assessments and of Some Possible Tools Supporting the CSM Regulation, ERA, Valenciennes Cedex, France, 06.01.2009, http://www.era.europa.eu. 